médecine/sciences $1986 ; 2: 193-7$

\title{
Hypersécrétion de I'hormone antidiurétique chez l'homme
}

Dans un état œdémateux avec hyponatrémie, on a pu montrer que les concentrations plasmatiques arginine-vasopressine (AVP) étaient élevées. Grâce à l'emploi d'antagonistes de cette hormone, il sera bientôt possible d'analyser le rôle de l'excès d'AVP dans diverses conditions cliniques et de traiter ces malades hyponatrémiques.

\section{Daniel Bichet Jean-Noël Barjon Vasiliki Papukna}

Service de néphrologie et centre de recherche. Hôpital du Sacré-Cour et Université de Montréal.

Remerciements

Daniel G. Bichet est boursier (" scholar ") de la Fondation canadienne des maladies du cœur. Ses études ont été subventionnées par la Fondation canadienne des maladies du cœur, la Fondation canadienne des maladies du rein, le Fonds de la recherche en santé du Québec et le Conseil de recherches médicales du Canada (MA-81 26).

\section{ADRESSE}

D. Bichet, J.-N. Barjon, V. Papukna : M. D., service de néphrologie et centre de recherche, Hôpital du Sacré-Cœur, 5400 ouest boulevard Gouin, Montréal (Québec) Canada $\mathrm{H}_{4} \mathrm{~J}_{\mathrm{IC}}$. a mesure de l'hormone antidiurétique humaine (arginine-vasopressine : AVP) par des dosages radioimmunologiques sensibles et spécifiques [I] a permis d'étudier de nombreuses entités physiopathologiques dans lesquelles une hypersécrétion d'AVP avait déjà été suspectée par des méthodes indirectes. Parallèlement, des études sur des fractions cellulaires utilisant des dérivés tritiés de la vasopressine et divers analogues structuraux ont défini deux classes principales de récepteurs spécifiques à l'AVP : des récepteurs vasculaires ou $V_{1}$ : presseurs, induisant après leur fixation une augmentation du calcium intracellulaire, vraisemblablement secondaire à l'hydrolyse des phosphoinositides membranaires; des récepteurs du tubule collecteur rénal ou $\mathrm{V}_{2}$ : antidiurétiques, couplés à l'adényl-cyclase [2]. La synthèse de très nombreux analogues structuraux de l'AVP par Manning et Sawyer [3] a permis l'étape fondamentale de la caractérisation pharmacologique des récepteurs à l'AVP et l'utilisation thérapeutique d'agonistes ainsi que, potentiellement, d'antagonistes de l'AVP.

Le but de cet article est d'analyser le rôle de l'hypersécrétion de l'AVP dans les états pathologiques bien définis chez l'homme, particulièrement dans les états œdémateux hyponatrémiques et dans les syndromes de sécrétion inappropriée d'ADH (SIADH), ainsi que de définir le rôle thérapeutique prometteur d'antagonistes spécifiques $\mathrm{V}_{2}$.

\section{Critères de I'hypersécrétion d'AVP}

Le déterminant principal de la libération d'AVP chez l'homme est l'osmolalité $[\mathbf{I}]$. Des variations très minimes de l'osmolalité plasmatique, de l'ordre de $\mathrm{i} \%$, sont perçues par des osmorécepteurs spécifiques de l'hypothalamus et induisent des variations minimes de l'AVP (de 0,5 à $5 \mathrm{pg} / \mathrm{ml}$ ) sécrétée par les noyaux hypothalamiques, supraoptique et paraventriculaire ( $f i g u$ re I). Très schématiquement, l'AVP libérée au niveau de l'hypophyse postérieure induit, par l'intermédiaire de récepteurs $\mathrm{V}_{2}$ du tubule collecteur, des modifications marquées de l'osmolalité urinaire : de 60 à I $200 \mathrm{mOsm} / \mathrm{kg} \mathrm{H}_{2} \mathrm{O}$. L'amplification de ce système est à souligner : des modifications minimes de l'AVP circulante induisent rapidement un état antidiurétique. L'hypersécrétion d'AVP doit donc 


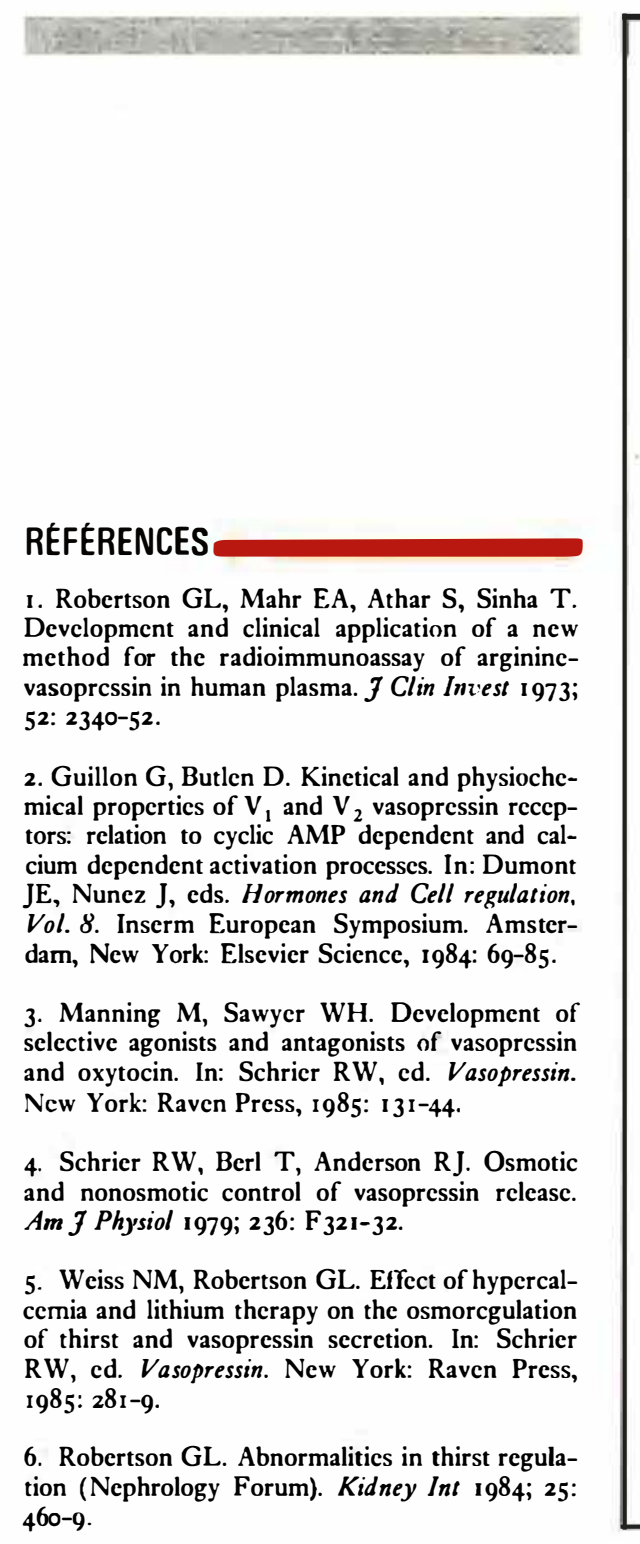

7. Bichet DG, van Putten VJ, Schrier RW Potential rolc of increased sympathetic activity in impaired sodium and water excretion in cirrhosis. $N$ Engl 7 Med 1982; 307: 1552-7.

8. Bichet DG, Kortas C, Mcttauer B., et al. Modulation of plasma and "platelet fraction" vasopressin and water excretion by cardiac function in patients with severe congestive heart failurc. Kidney' Int 1986 (sous presse).

9. Bichet DG, Groves BM, Schrier RW. Mechanisms of improvement of water and sodium excretion by immersion in decompensated cirrhotic patients. Kidney Int 1983; 24: 788-94.

ı. Grimaldi A, Pruszczynski W, Therbert F, Ardaillou R. Antidiuretic hormone response to volume depletion in diabctic patients with cardiac autonomic dysfunction. Clin $\mathrm{S}_{c} i$ 1 985; 68: 545-52.

I1. Thamcs MD, Pctcrson MG, Schmid PG. Stimulation of cardiac receptors with veratrum alkaloids inhibits ADH secretion. Am $\mathcal{Y}$ Physiol 1980; 239: $\mathrm{H}_{7} 84-8$

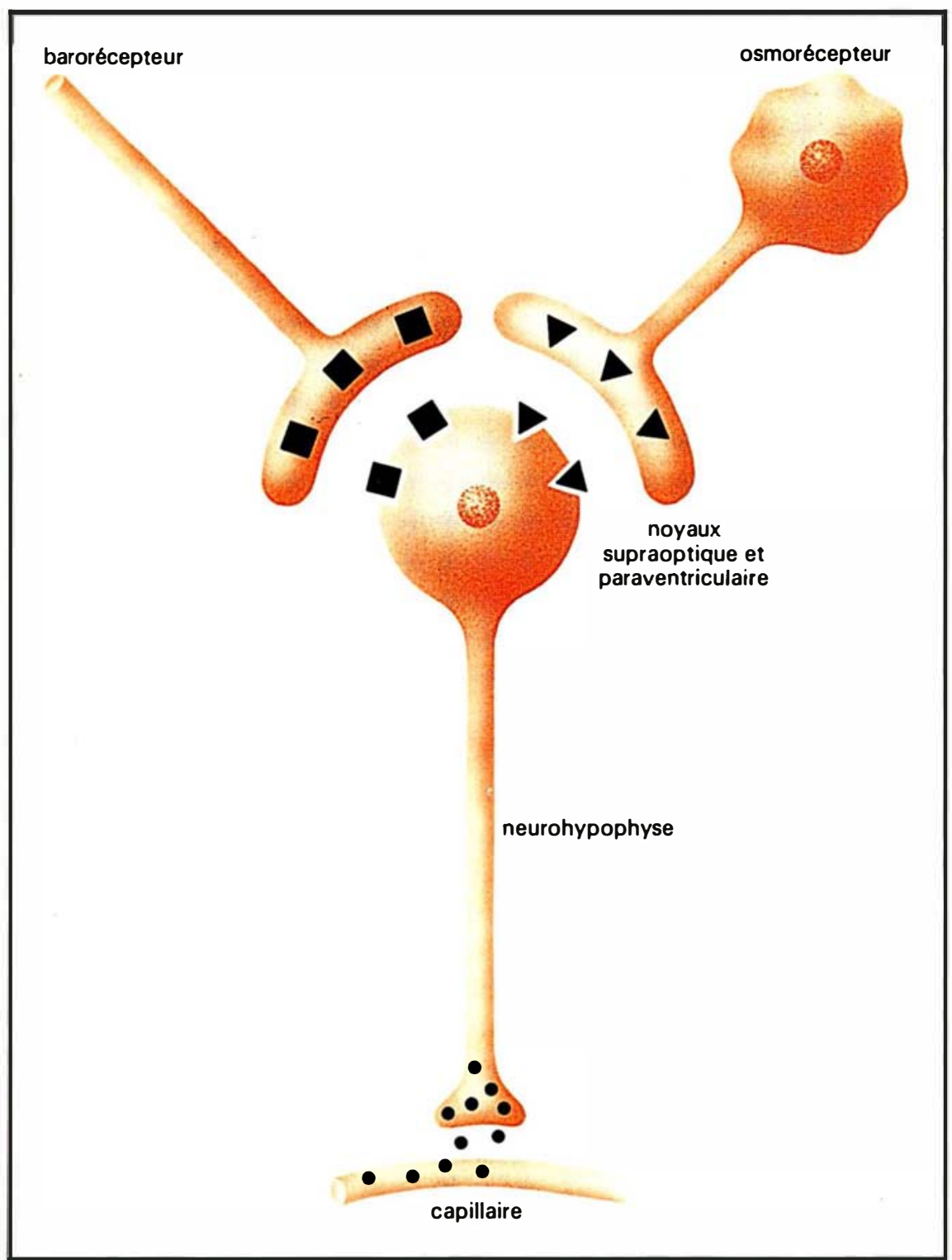

Figure 1. Représentation schématique du contrôle osmotique et nonosmotique de la sécrétion de la vasopressine.

être définie en fonction de la mesure concomitante de l'osmolalité ou du sodium plasmatique (figure 2). Un état d'hypersécrétion sera présent quand l'AVP mesurée sera plus élevée que les niveaux prévus par le nomogramme. L'hypersécrétion d'AVP peut être osmotique ou non-osmotique (figure I). En effet, les cellules magnocellulaires reçoivent des influx provenant des osmorécepteurs mais aussi des influx afférents de barorécepteurs ou d'autres structures mal définies responsables de la stimulation de l'AVP par l'hypoglycémie ou par les réflexes de nausée [4]. Ainsi, la diminution de la pression artérielle, l'hémorragie, la constriction de la veine cave inférieure s'accompagnent-elles d'une augmentation de l'AVP circulante par l'intermédiaire d'afférences provenant de barorécepteurs situés sur le circuit à haute pression. Ces facteurs non-osmotiques sont responsables de la majorité des états d'hypersécrétion d'AVP identifiés chez l'homme. A l'opposé, l'hypersécrétion osmotique de l'AVP, c'est-à-dire l'augmentation de la pente ou sensibilité (figure 2) de la libération de l'AVP, en réponse à la stimulation osmotique, est une entité rare décrite seulement 
dans les cas de diabètes insipides néphrogéniques secondaires au lithium ou au calcium [5] ou dans les diabètes insipides néphrogéniques congénitaux (observations personnelles).

L'hypersécrétion d'AVP peut être appropriée ou inappropriée. Les stimulations osmotiques et non-osmotiques sont approprićes. Ainsi, l'augmentation de l'AVP après hémorragic, suite à la diminution de la pression artérielle et du volume circulant artériel efficace (VCAE) perçue au niveau des barorécepteurs artériels, doit être considérée comme appropriée : l'AVP serait libérée pour ses propriétés vasoconstrictrices et antidiurétiques. A l'opposé, la libération d'AVP par des cellules tumorales anaplasiques, sans aucune réponse à l'osmolalité, est inappropriée.

Finalement, toute hypersécrétion d'AVP doit être analysée en fonction des autres facteurs responsables de l'homéostasie hydrique, c'est-àdire la soif et les facteurs intrarénaux qui permettent la formation d'urine concentrée ou diluée. Ainsi, l'hypersécrétion d'AVP s'accompagne habituellement d'une sensation de soif, stimulée par des mécanismes osmotiques et non-osmotiques parallèles à ceux décrits précédemment [6]. L'augmentation de la prise d'eau et l'hypersécrétion d'AVP sont alors responsables de l'hyponatrémie. Dans les états œdémateux, la diminution du VCAE induit en outre une diminution de la perfusion rénale. Cet effet intrarénal limite la quantité d'eau et de solutés présentée au segment de dilution du néphron et diminue ainsi l'excrétion de l'eau libre. Ces facteurs intrarénaux et la stimulation nonosmotique de l'AVP (facteur extrarénal) contribuent au défaut de l'excrétion de l'eau observée.

\section{États odémateux hyponatrémiques}

Étudions à présent l'hypersécrétion d'AVP dans les états œdémateux hyponatrémiques : la cirrhose hépatique décompensée et l'insuffisance cardiaque congestive sévère. Nous avons récemment mesuré des taux plasmatiques élevés d'AVP chez des patients hyponatrémiques avec cirrhose hépatique décompensée [7] ou insuffisance cardiaque sévère [8] (figure 2). Des patients normaux, sans maladie hépatique, cardiaque ou rénale, excrètent rapidement $(<4 \mathrm{~h})$ et complètement $(>80 \%)$ une charge en eau de $20 \mathrm{ml} / \mathrm{kg}$ de poids corporel. Les patients cardiaques ont excrété $3 \mathrm{I} \%$ et les patients cirrhotiques $30 \%$ de la charge en eau administrée au cours des cing heures suivantes et une corrélation inverse fut observée entre l'AVP et le pourcentage de la charge en eau excrétée. Chez les patients insuffisants cardiaques, la clairance de la créatinine était identique dans le groupe avec AVP supprimée, comparé au groupe avec hypersécrétion d'AVP. Cependant, dans ces deux groupes, le pourcentage d'excrétion de l'eau était très différent, soulignant ainsi la prépondérance des facteurs extrarénaux (stimulation non-osmotique de l'AVP) vis-à-vis des facteurs intra- rénaux dans le défaut d'excrétion de l'eau observé. Les marqueurs du VCAE (activité rénine plasmatique, aldostérone, noradrénaline plasmatique) furent trouvés plus élevés chez les patients cardiaques et cirrhotiques avec hypersécrétion d'AVP. De plus, l'amélioration du VCAE par l'immersion chez huit patients cirrhotiques non-excrétcurs amena une suppression de l'AVP circulante et une amélioration de l'excrétion de l'eau [9]. Dans l'insuffisance cardiaque, l'amélioration de l'hémodynamique cardiaque par les vasodilatateurs s'est accompagnée d'une diminution de l'AVP et d'une augmentation de l'excrétion de l'eau [8]. Dans la cirrhose hépatique décompensée, l'augmentation de la capacitance du territoire splanchnique, la vasodilatation périphérique et l'ascite contribueraient à une diminution du VCAE perçue au niveau des barorécepteurs artériels avec stimulation réflexe de l'AVP $[7,9]$. Dans l'insuffisance car-

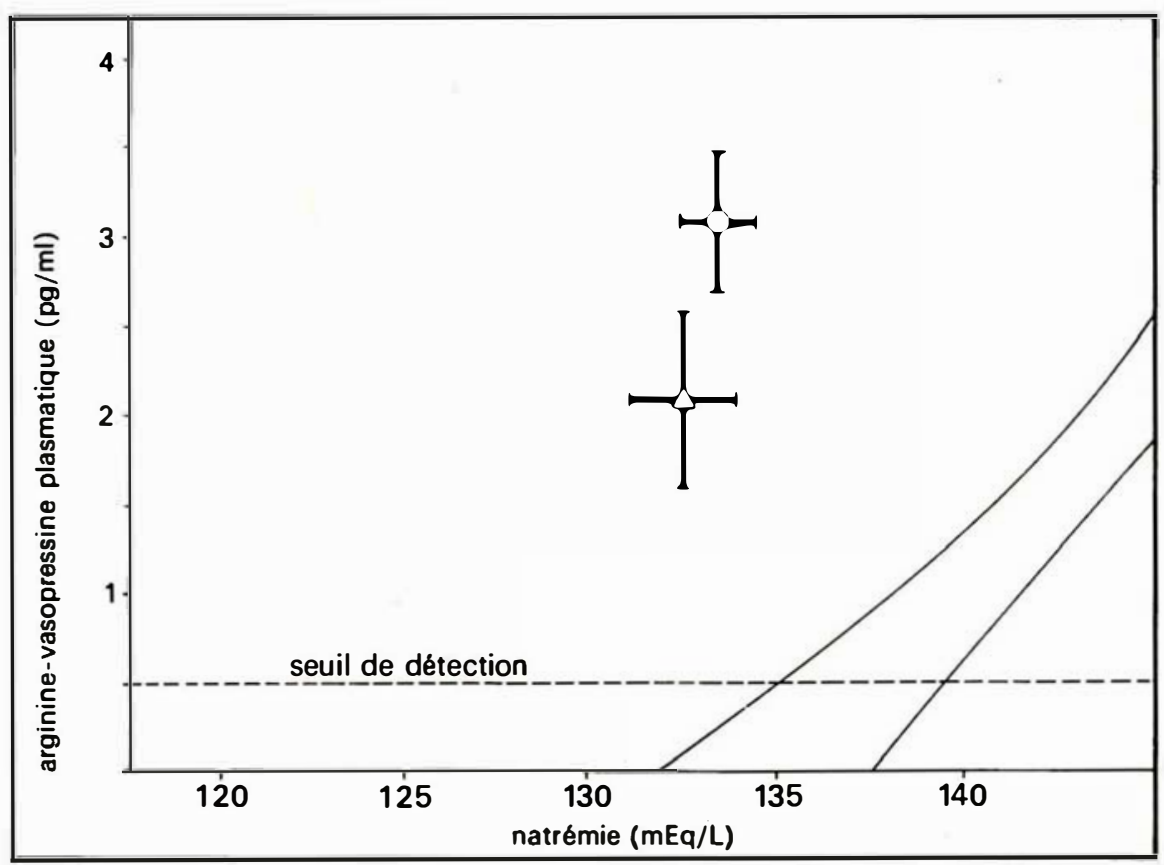

Figure 2. Définition de l'hypersécrétion de l'hormone antidiurétique suivant l'intervalle de confiance 99 \% natrémie/arginine-vasopressine plasmatique établi pour 19 individus normaux au cours de tests de charge en eau et perfusion de salin hypertonique (zone grise). La surface rosée représente les états d'hypersécrétion. Des patients hyponatrémiques avec cirrhose décompensée $(\triangle)$ ou avec insuffisance cardiaque $(O)$ sont représentés $[7,8]$. 
diaque, nos données comme celles de Grimaldi et coll. [1o] favoriseraient l'importance des récepteurs intraventriculaires cardiaques modulant la libération d'AVP [II].

\section{SAl et SIADH}

Le diagnostic de syndrome d'antidiurèse inappropriée (SAI) peut être évoqué en présence d'une hyponatrémie chez un patient avec des fonctions hépatique, cardiaque, rénale, corticosurrénalienne et thyroïdienne normales et en l'absence de médicaments reconnus comme pouvant altérer la libération de l'AVP ou l'excrétion d'eau par le rein [12]. Si des niveaux inappropriés d'AVP sont mesurés, on peut alors établir le diagnostic de syndrome de sécrétion inappropriée d'hormone antidiurétique (SIADH). Dans la plupart des cas, SAI et SIADH vont de pair. Cependant, d'après Zerbe et Robertson [12], dans plus de dix pour cent des cas de SAI, l'AVP immunoréactive circulante est indétectable ou normale (SIADH dits de type IV). Pour ajouter à la confusion, il n'existe aucune corrélation entre les différents types de SIADH décrits et la maladie sous-jacente [12]. La description physiopathologique et le traitement des différents types de SIADH pourrait être améliorés par l'utilisation d'antagonistes spécifiques de l'AVP.

\section{Agonistes et} antagonistes de l'AVP

De nombreuses études expérimentales utilisant des agonistes spécifiques $\mathrm{V}_{1}$ ont examiné le rôle possible, central et périphérique de l'AVP dans la régulation de la pression artérielle [13] (figure 3). L'hémorragie, la déshydratation, la déplétion de volume plasmatique

13. Liard JF. Vasopressin in cardiovascular control: role of circulating vasopressin. Clin $\mathrm{Sci}$ 1984; 67: 473-81.

14. Stassen FL, Bryan W, Gross M, et al. Critical differences between species in the in vivo and in vitro renal responses to antidiuretic hormone antagonists. In: Cross BA, Leng G, eds. The neurohypophysis: structure, function and control, Progress in Brain Research. Amsterdam, New York: Elsevier Science 1983; 60: 395-403.

15. Manning M, Olma A, KIis W, et al. Carboxy terminus of vasopressin required for activity but not binding. Nature 1984; 308: 652-3.

16. Kim JK, Dillingham MA, Summer SN, Ishikawa S, Anderson RJ, Schrier RW. Effects of vasopressin antagonist on vasopressin binding, adenylate cyclase activation and water flux. $7 \mathrm{Clin}$ Invest $1985 ; 76$ : $1530-5$.

17. Ishikawa $S$, Kim JK, Schrier RW. Further in vivo evidence for antagonist to hydroosmotic action of arginine-vasopressin. Am $\mathcal{J}$ Physiol 1983; 245: R713-9.

18. Kinter LB, Dytko G, McDonald J, Huff man $W$, Stassen F. Prevention of hyponatremia in experimental Schwartz-Bartter syndrome with a vasopressin antagonist (SK\&F 101926). Kidney Int 1986; 29: 418.

19. Ishikawa S, Saito P, Okada K, et al. Effect of vasopressin antagonist on renal water excretion in rats with inferior vena cava constriction. Kidney Int (sous presse)

20. Yared A, Kon V, Brenner BM, Ichikawa I. Role of vasopressin in rats with congestive heart failure. Kidney Int 1985; 27 : 337.

21. Nicod P, Waeber B, Bussien JP, et al. Acute hemodynamic effect of a vascular antagonist of vasopressin in patients with congestive heart failure. Am 7 Cardiol 1 985; 55: 1043-6.

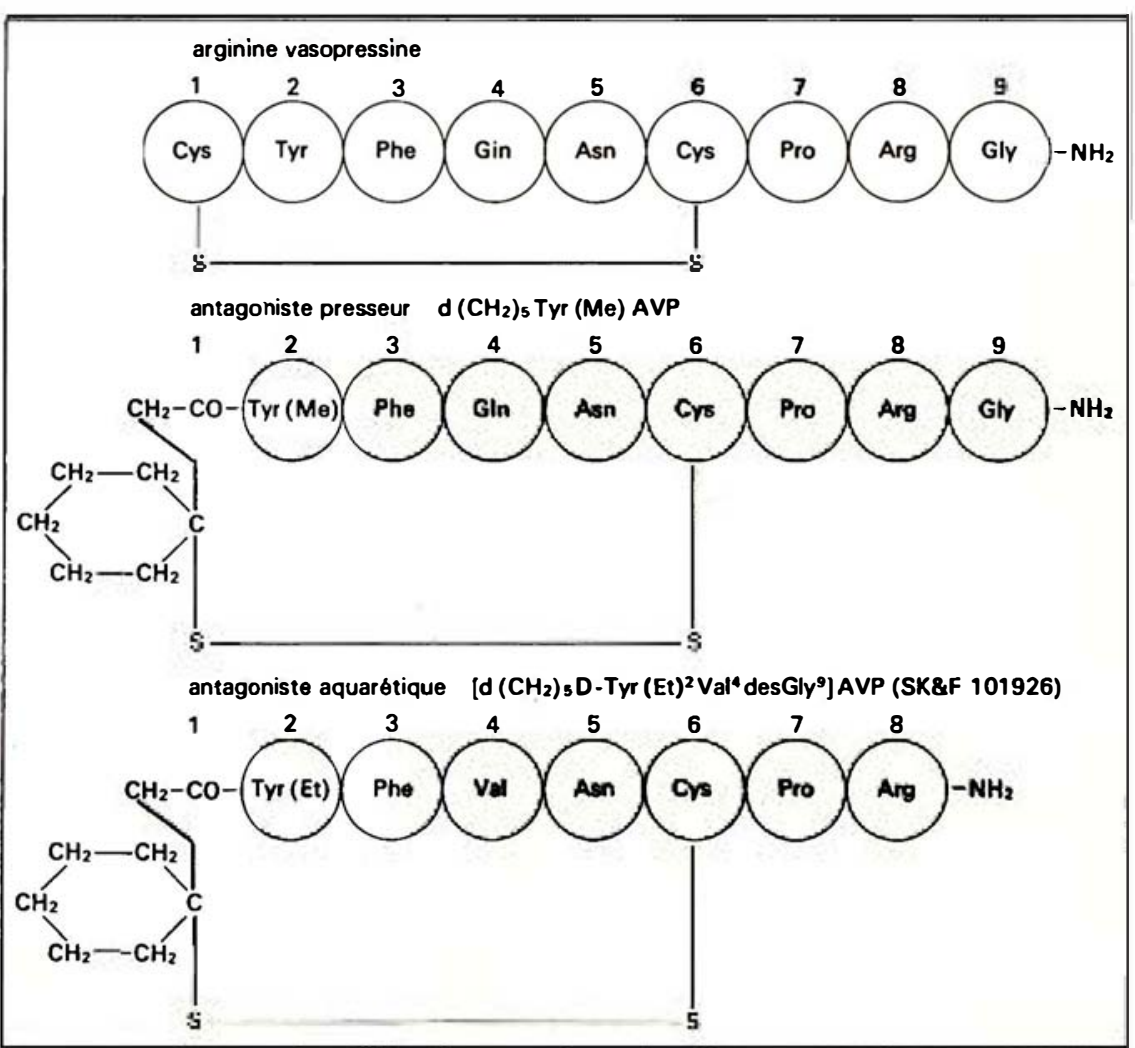

Figure 3. Structures de l'arginine-vasopressine, d'un antagoniste presseur et d'un antagoniste aquarétique. 


\section{Caractéristiques des antagonistes « aquarétiques »}

In vitro

I) Entrent en compétition spécifique avec l'AVP tritiée pour les mêmes sites de liaison [I6].

2) Inhibent l'activation de l'adényl cyclase par l'AVP au niveau des tubules collecteurs corticaux, médullaires et papillaires ainsi qu'au niveau du segment large ascendant médullaire [i6].

3) Ne modifient pas la stimulation de l'adényl cyclase rénale secondaire à l'hormone parathyroïdienne, au glucagon et à la calcitonine [I6].

4) Inhibent le transport net d'eau secondaire à l'AVP à travers l'épithélium de tubules collecteurs isolés et perfusés [16].
In vivo

I) Diminuent l'augmentation de l'osmolalité urinaire secondaire à l'AVP endogène ou bien exogène [17].

2) $\mathrm{Ne}$ modifient ni l'excrétion des solutés, ni la filtration glomérulaire, ni la pression artérielle moyenne [ $\mathrm{r}$ ]

3) Préviennent l'apparition de I'hyponatrémie et de ses manifestations cliniques dans un modèle de SIADH induit chez le rat après administration chronique d'eau et d'AVP [18], ainsi que la rétention d'eau dans deux modèles expérimentaux d'insuffisance cardiaque $[19,20]$. secondaire aux diurétiques et l'insuffisance surrénalienne s'accompagnent d'une augmentation modérée de l'AVP secondaire à la diminution de VCAE. Dans ces conditions, la sécrétion d'AVP apparaît comme un mécanisme de défense à court terme contre l'hypotension et l'hypovolémie. A l'opposé, le rôle de l'AVP dans l'hypertension artérielle humaine est encore très mal connu.

Des antagonistes $V_{2}$ chez le rat se sont avérés agonistes chez le chien, suggérant une certaine hétérogénéité des récepteurs chez les mammifêres [14]. Des données récentes ont établi que ni le radical carboxyl ni le radical aminé ne sont nécessaires à la liaison de l'AVP à son récepteur $V_{1}$ ou $V_{2}$ mais ils sont par contre indispensables à leur activation [15]. Ainsi, l'antagoniste $\mathrm{V}_{2}$ décrit sur la figure 3 se lie au récepteur mais ses modifications structurales inhibent le déclenchement de la réponse cellulaire. Les caractéristiques de ces antagonistes $\mathrm{V}_{2}$ : anti-antidiurétiques ou "aquarétiques " [14] sont décrites dans l'encadré ci-dessus et leur utilisation dans les états avec hypersécrétion
d'AVP est imminente. Dans l'insuffisance cardiaque ou hépatique, l'utilisation d'aquarétiques pourra permettre d'une part une définition plus précise des facteurs intrarénaux responsables de l'hyponatrémie et d'autre part permettre une meilleure utilisation des médicaments natriurétiques classiques. Dans l'insuffisance cardiaque humaine, l'utilisation d'un antagoniste $V_{1}$ ne s'est accompagnée que très rarement d'une diminution des résistances vasculaires périphériques et de la post-charge [2I].

En conclusion, hormis les cas de SIADH, les états d'hypersécrétion de l'AVP sont la conséquence d'une diminution du VCAE avec stimulation non-osmotique de la vasopressine. L'amélioration du VCAE diminue l'hypersécrétion d'AVP. Dans ces conditions cliniques fréquentes, l'hypersécrétion d'AVP et un excès minime à modéré de l'eau infusée ou ingérée, conduisent à un état hypo-osmolaire parfois symptomatique. Dans ces cas, l'utilisation d'antagonistes spécifiques aquarétiques pourrait s'avérer un outil précieux d'investigation et de traitement

\section{Summary}

Accurate measurements of plasma arginine-vasopressin (AVP) have helped to define some vasopressin excess conditions in humans, specifically hyponatremic edematous states: decompensated liver cirrhosis and congestive heart failure, and the syndrome of inappropriate antidiuretic hormone secretion. In hyponatremic edematous states, a decrease in effective arterial blood volume (EABV) induced a non-osmotic stimulation of AVP; maneuvers which improved $E A B V$ decreased AVP and improved water excretion. Also, in vitro binding studies using agonists and antagonists of AVP have characterized $\mathrm{V}_{1}$ : pressor, and $\mathrm{V}_{2}$ : antidiuretic specific receptors for AVP. Finally, synthetic $\mathrm{V}_{2}$ antagonists are potential investigation and therapeutic tools in humans with vasopressin hypersecretion.

\section{TIRES A PART}

D. Bichet : M. D., service de néphrologic et centre de recherche, Hôpital du Sacré-Cœur, 5400 oucst boulcvard Gouin, Montrćal (Québec) Canada $\mathrm{H}_{4} \mathrm{~J}_{\mathrm{IC}}$. 